\title{
Padrão de mortalidade por câncer em trabalhadores eletricitários
}

\author{
A cancer mortality pattern in Brazilian \\ electrical workers
}

Inês E. Mattos 1

Naim Sauaia 2

Paulo R. Menezes 2

\footnotetext{
1 Departamento de Epidemiologia e Métodos Quantitativos em Saúde, Escola Nacional de Saúde Pública, Fundação Oswaldo Cruz. Rua Leopoldo Bulhões 1480, 8 o andar, Rio de Janeiro, $R J$ 21041-210, Brasil.

2 Departamento de Medicina Preventiva, Faculdade de Medicina, Universidade de São Paulo. Av. Dr. Arnaldo 455, São Paulo, SP 01246-903, Brasil.
}

\begin{abstract}
This study provides the cancer mortality pattern for a cohort of 10,017 electric power plant workers from 1978 to 1994. Standard mortality ratio (SMR) analysis showed lower mortality from all causes (0.47: 0.43-0.51) and from all groups of causes including neoplasms (0.73: 0.60-0.90) among these workers, in comparison with the same sex and age bracket in Rio de Janeiro. In relation to specific cancer sites, standard cancer mortality ratios (SCMRs) greater than one were observed for neoplasms of the small bowel (10.35: 3.34-32.09), gallbladder (2.64: 0.99-7.03), pancreas (1.83: 0.91-3.66), kidney (2.91: 1.39-6.10), and bladder (2.60: 0.58-4.12), as well as for melanoma (1.82: 0.46-7.28). Important limitations were the workers' relatively young age and the short period of time since job admission (less than 15 years). The small number of deaths for each anatomic site led to instability in the study results, with many non-significant SCMRs and wide confidence intervals. Due to these limitations, study results are difficult to interpret in light of currently available evidence.
\end{abstract}

Key words Electromagnetic Fields; Neoplasms; Occupational Exposure; Cohort Studies

Resumo O trabalho analisa a mortalidade por câncer de uma coorte de 10.017 trabalhadores de empresa geradora de eletricidade, no período 1978-1994. A análise das Razões Padronizadas de Mortalidade mostrou que os eletricitários apresentam menor mortalidade geral $(0,47(0,43-$ 0,51)) e pelos diferentes grupos de causas de morte, inclusive neoplasmas $(0,73(0,60-0,90))$, em comparação com a população do mesmo sexo e faixa etária do Rio de Janeiro. Foram observadas Razões Padronizadas de Mortalidade por Câncer maiores do que a unidade para neoplasias de intestino delgado (10,35 (3,34-32,09)); vesícula biliar (2,64 (0,99-7,03)); pâncreas (1,83 (0,913,66)); rim (2,91 (1,39-6,10); bexiga (2,60 (0,58-4,12) e melanoma (1,82 (0,46-7,28). Uma importante limitação do estudo é a coorte ser constituída por indivíduos jovens, com menos de 15 anos de serviço. O número limitado de óbitos por localização anatômica não propiciou estabilidade aos resultados das análises estatísticas, evidenciando-se em alguns intervalos de confiança amplos e sem significância estatística. Devido às limitações, os resultados obtidos são de difícil interpretação, à luz do conhecimento disponível.

Palavras-chave Campos Eletromagnéticos; Neoplasias; Exposição Ocupacional; Estudo de Coortes 


\section{Introdução}

No Brasil, o processo de industrialização ocorreu de maneira rápida, não proporcionando tempo suficiente para adaptação das instalações industriais, treinamento da força de trabalho, estabelecimento de serviços de saúde ocupacional eficientes e de legislação trabalhista adequada. Em nosso meio, existem poucas informações sobre exposições ocupacionais e condições de trabalho. Alguns trabalhos publicados, entretanto, apontam para níveis elevados de contaminação e condições insatisfatórias de trabalho (Fernicola et al., 1976; Kato et al., 1993), e estudos epidemiológicos, realizados com grupos de trabalhadores industriais brasileiros, têm evidenciado um maior risco de desenvolvimento de neoplasias associado com essa atividade (Mattos \& Koifman, 1996; Wünsch Filho et al., 1995).

Alguns autores acreditam que o impacto dos agentes cancerígenos utilizados na produção industrial seja ainda mais acentuado sobre a saúde dos trabalhadores dos países periféricos, pois a maioria das indústrias e ocupações que acarretam um risco importante de desenvolvimento de câncer estão presentes e, algumas vezes, parecem utilizar processos de produção considerados obsoletos nos países desenvolvidos, ocasionando um maior grau de poluição (Vainio et al., 1994).

Em 1982, Milham Jr. levantou a possibilidade de que a exposição ocupacional aos campos eletromagnéticos pudesse estar relacionada ao desenvolvimento de neoplasias. Estudando a mortalidade proporcional de homens residentes no Estado de Washington (Estados Unidos), segundo causa e ocupação, o referido pesquisador observou um aumento dos óbitos por leucemia entre aqueles cuja atividade profissional envolvia níveis elevados de exposição aos campos eletromagnéticos (Milham Jr., 1982).

A partir desse trabalho, diversos estudos epidemiológicos analisaram as relações entre a exposição ocupacional a campos eletromagnéticos e o desenvolvimento de câncer. Uma série de estudos evidenciou um maior risco de desenvolvimento de leucemias para trabalhadores do setor elétrico (Bastuji-Garin et al., 1990; Flodin et al., 1986; Milham Jr., 1985; Stern et al., 1986; Thériault et al., 1994). Outros estudos, todavia, não observaram resultados positivos (Sahl et al., 1993; Savitz \& Loomis, 1995; Schreiber et al., 1993). Uma pesquisa de mortalidade proporcional por câncer (PMR) em funcionáros de uma companhia geradora de eletricidade em São Paulo também não observou um excesso de óbitos por leucemia en- tre aqueles trabalhadores (Mattos \& Koifman, 1996).

Diversos pesquisadores observaram uma associação positiva entre a exposição ocupacional aos campos eletromagnéticos e o desenvolvimento de câncer de cérebro (Grayson, 1996; Lin et al., 1985; Pearce et al., 1989; Savitz \& Loomis, 1995; Thomas et al., 1987; Tynes et al., 1992), enquanto, em outros estudos, essa associação não foi evidenciada (Sahl et al., 1993; Savitz \& Loomis, 1995; Schreiber et al., 1993; Tynes et al., 1994). No Brasil, no estudo de mortalidade anteriormente referido, foi observado um maior número de óbitos por câncer de cérebro entre os trabalhadores com exposição provavelmente elevada aos campos magnéticos (Mattos \& Koifman, 1996).

Estudos de coortes ocupacionais têm mostrado associação entre a exposição a campos eletromagnéticos e o desenvolvimento de melanomas (Olin et al., 1985; Vagero et al., 1985). Thériault et al. (1994), em um estudo caso-controle aninhado com trabalhadores eletricitários, não observaram associação entre a exposição a campos magnéticos e o desenvolvimento desse tumor.

A ocorrência de linfomas também foi relacionada à exposição ocupacional a campos eletromagnéticos (Savitz et al., 1993).

Na literatura, existem poucas evidências de uma associação entre exposição a campos eletromagnéticos e o desenvolvimento de tumores do aparelho urinário. Em um estudo de coorte realizado na Suécia, Tornqvist et al. (1986) observaram um aumento do risco de desenvolver esse tipo de câncer entre operadores de usinas geradoras de eletricidade e eletricistas de linhas de transmissão. Estudando a mortalidade proporcional por câncer em trabalhadores de uma companhia geradora de eletricidade de São Paulo, Mattos \& Koifman (1996) observaram um aumento do número de óbitos por câncer de bexiga, estatisticamente significativo, no grupo de trabalhadores com exposição provavelmente alta aos campos magnéticos.

A disponibilidade de dosímetros para medir a exposição aos campos elétricos e magnéticos proporcionou a incorporação de medidas diretas de exposição aos estudos epidemiológicos, permitindo uma caracterização mais precisa dos grupos expostos e a avaliação de diversos parâmetros de exposição (Savitz et al., 1993). As pesquisas mais recentes, com um desenho de estudo mais cuidadoso, sob o ponto de vista metodológico, utilizaram essas medidas para analisar grandes grupos de trabalhadores do setor elétrico, mas, mesmo assim, ainda não foi possível definir, de maneira conclu- 
siva, a natureza dessa associação (Sahl et al., 1993; Savitz \& Loomis, 1995; Thériault et al., 1994) (Tabela 1).

As bifenilas policloradas (PCBs) são substâncias que, em virtude de sua grande estabilidade química e térmica, têm grande utilização como fluidos dielétricos em transformadores e capacitores elétricos (Ouw et al., 1976). Com base em evidências de estudos realizados em animais, esse agente químico foi considerado pelo International Agency for Research on Cancer (IARC), como potencialmente cancerígeno para seres humanos (Vainio et al., 1994). Os trabalhadores de fábricas produtoras de transformadores e capacitores elétricos, assim como os eletricitários que atuam na manutenção e substituição desses equipamentos estão expostos a essa substância (Phillips et al., 1989). Embora a utilização desse agente químico esteja proibida em diversos países, os equipamentos elétricos antigos continuam em funcionamento, até sua gradual substituição por novos modelos (Sinks et al., 1992).

Em relação a PCBs, são muito poucos os estudos epidemiológicos disponíveis na literatura e seus resultados não são consistentes, e nenhum deles analisou a ocorrência de câncer em trabalhadores eletricitários (Tabela 1). As neoplasias que têm sido associadas a essa exposição são os tumores de fígado, de vesícula biliar, de cérebro e melanomas (Brown, 1987; Sinks et al., 1992). Dois estudos epidemiológicos recentes analisaram a exposição a esse agente químico como uma variável de confundimento da associação entre campos magnéticos e câncer, concluindo que não houve modificação dos resultados, ao controlar por possível exposição a PCBs (Savitz \& Loomis, 1995; Thériault et al., 1994).

O contato com diferentes tipos de solventes também é freqüente no ambiente de trabalho do setor elétrico (Savitz et al., 1993; Savitz \& Loomis, 1995; Thériault et al., 1994). Alguns estudos epidemiológicos com outros grupos ocupacionais, como trabalhadores das indústrias da borracha e petroquímica, mostraram evidências de uma associação entre exposição a solventes e o desenvolvimento de leucemia e câncer de cérebro (Lazarov et al., 2000; Satin et al., 1996) (Tabela 1). Os trabalhos de Thériault et al. (1994) e de Savitz \& Loomis (1995) analisaram a exposição a solventes como uma possível variável de confundimento da associação entre campos magnéticos e neoplasias, não observando modificação nos resultados obtidos, ao controlar por potencial exposição a esse grupo de produtos químicos.

Tabela 1

Estudos epidemiológicos sobre a associação entre fatores de risco presentes no ambiente de trabalho do setor elétrico e o desenvolvimento de neoplasias.

\begin{tabular}{|c|c|c|c|}
\hline Referência & Tipo de estudo & Neoplasias & Resultados \\
\hline \multicolumn{4}{|l|}{ Campos eletromagnéticos } \\
\hline \multirow[t]{2}{*}{ Sahl et al. (1993) } & Caso-controle aninhado & Leucemias & OR $1,01(0,75-1,36)$ \\
\hline & & Cérebro & OR $0,95(0,36-2,44)$ \\
\hline \multirow[t]{2}{*}{ Savitz \& Loomis (1995) } & Coorte & Leucemias & RR $1,13(0,70-1,82)$ \\
\hline & & Cérebro & RR 1,47 (0,84-2,56) \\
\hline \multirow[t]{2}{*}{ Thériault et al. (1994) } & Caso-controle aninhado & Leucemias & OR 1,54 $(0,90-2,63)$ \\
\hline & & Cérebro & OR 1,54 (0,85-2,81) \\
\hline \multicolumn{4}{|c|}{ Bifenilas policloradas (PCBs) } \\
\hline \multirow[t]{2}{*}{ Sinks et al. (1992) } & SMR & Melanoma & SMR 4,1 $(1,8-8,0)$ \\
\hline & & Cérebro e sistema nervoso & SMR $1,8(0,6-4,2)$ \\
\hline Brown et al. (1987) & SMR & Fígado e vesícula biliar & SMR 2,8 (não apresentado) \\
\hline \multicolumn{4}{|l|}{ Solventes } \\
\hline Satin et al. (1996) & SMR & Sistema nervoso central & SMR 1,1 $(7,9-15,1)$ \\
\hline Lazarov et al. (2000) & Caso-controle & Leucemia mielóide aguda & OR 2,52 $(1,45-4,39)$ \\
\hline
\end{tabular}

$\mathrm{OR}=$ odds ratio; $\mathrm{RR}=$ risco relativo; $\mathrm{SMR}$ = razão padronizada de mortalidade 


\section{Material e métodos}

O presente trabalho consiste em um estudo de coorte histórica de trabalhadores de uma empresa de produção e transmissão de energia elétrica no Rio de Janeiro. O período do estudo está compreendido entre 1o de janeiro de 1978 e 31 de dezembro de 1994, abrangendo 17 anos.

Eram elegíveis para o estudo todos os trabalhadores do sexo masculino que, na data de início do estudo, haviam completado pelo menos cinco anos de trabalho na empresa; os demais trabalhadores do mesmo sexo, já admitidos naquela data, à medida que fossem completando esse tempo de serviço, e aqueles admitidos após 1o de janeiro de 1978, a partir do momento em que completavam cinco anos de atividade na empresa, desde que essa data fosse anterior à data de término do estudo.

A opção pelo estabelecimento de um período mínimo de cinco anos de serviço na empresa para ingresso na coorte está relacionada a aspectos ligados à atuação dos agentes cancerígenos no processo de desencadeamento do câncer e a razões operacionais que visavam aumentar a possibilidade de seguimento dos trabalhadores, para determinação da sua condição vital ao término do estudo. Isso evita a inclusão de trabalhadores temporários, que costumam apresentar padrões de mortalidade diferentes daqueles dos trabalhadores estáveis (Lin et al., 1985; Steenland \& Stayner, 1991; Wertheimer \& Leeper, 1982; Wilcosky \& Wing, 1987).

A identificação dos trabalhadores da coorte foi realizada através da folha de pagamento do departamento de pessoal da empresa, sendo utilizado o mês de dezembro de cada ano do período de estudo. Foram impressas relações que continham nome do trabalhador, local de trabalho, data de nascimento, data de admissão, função, data e motivo de saída, quando fosse o caso.

A condição vital de cada trabalhador da coorte, na data do término do período de seguimento, foi determinada por meio de fontes de informação da empresa. Para os trabalhadores ativos, essa fonte de informação foi a folha de pagamento de janeiro de 1995, sendo considerados vivos aqueles que se encontravam em atividade naquela data. Para os trabalhadores que haviam deixado a empresa, a determinação da condição vital foi feita com base no cadastro de contribuintes da sociedade de previdência privada da empresa, através de uma relação nominal de todos os contribuintes de sexo masculino do mês de dezembro de 1995. Foram considerados vivos todos os trabalhadores cujos nomes constavam dessa relação, sendo utilizada a data do nascimento para confirmar, em cada caso, se o indivíduo em questão era efetivamente um dos membros da coorte. Quando a condição vital do trabalhador não pôde ser determinada, o mesmo foi considerado vivo até a data de saída da empresa, momento em que terminou o seu período de seguimento (Checkoway et al., 1989).

As certidões de óbito dos trabalhadores foram procuradas nos arquivos da sociedade de previdência privada, que mantém arquivo com cópias das certidões para todos os contribuintes falecidos. As causas de morte mencionadas na certidão de óbito de cada trabalhador foram transcritas e, posteriormente, codificadas por médico com experiência em codificação de doenças, de acordo com as normas da 9a Revisão da Classificação Internacional de Doenças (OMS, 1985), sendo cada óbito classificado, de acordo com a causa básica de morte, em um dos grupos de doenças da Lista de Três Algarismos. As neoplasias foram codificadas utilizando-se três dígitos, seguindo os mesmos procedimentos. Os óbitos cujas certidões não foram localizadas (dois casos) foram incluídos no grupo de causas mal definidas (Checkoway et al., 1989).

Em relação à determinação da exposição ocupacional, os dados disponíveis na empresa são bastante limitados, não existindo nenhum tipo de quantificação. Entretanto, estudos de medição de campos elétricos e magnéticos confirmaram que certas ocupações do setor elétrico têm, efetivamente, uma exposição elevada a esses agentes (Koifman \& Thériault, 1994). Quanto a PCBs, informações de técnicos da empresa dão conta de que esse produto era utilizado em todos os transformadores e capacitores até 1985, data de sua proibição no Brasil, sendo, então, substituído por outro produto químico nos novos equipamentos. Continuam presentes, contudo, nos transformadores antigos ainda em uso. Os técnicos confirmam a utilização de diferentes tipos de solventes no setor elétrico, sendo, em anos mais recentes, abolido o uso do benzeno, obedecendo a normas legais. Outros solventes continuam sendo utilizados.

As variáveis que constavam das relações de folhas de pagamento foram utilizadas para construção do banco de dados do estudo, com o programa dBASE III Plus, versão 1.1 (Borland International, 1986), sendo agregadas, posteriormente, as informações sobre a condição vital dos trabalhadores e, em caso de óbito, a data deste e a causa básica de morte. Os procedimentos de análise do banco de dados, para obtenção dos resultados deste estudo, foram realizados com os programas Epi Info 6, versão 
6.04 (CDC/WHO, 1996) e Stata 5.0 (Stata Corporation, 1997)

Na primeira parte desse trabalho, devido à escassez de dados que possibilitassem avaliar melhor a exposição, o conjunto de eletricitários foi considerado como grupo ocupacionalmente exposto aos fatores de risco, alvo do estudo. A mortalidade por grupos de causa de morte dos trabalhadores eletricitários foi comparada com as taxas de mortalidade da população de referência, para obtenção das Razões Padronizadas de Mortalidade (SMRs), geral e por grupos de causas de morte, ajustadas por faixa etária e períodos de anos-calendário. $\mathrm{Pa}$ ra cada SMR, foi calculado um intervalo de confiança de 95\% (Checkoway et al., 1989). A seguir, a mortalidade pelas diferentes localizações anatômicas de neoplasias dos membros da coorte foi comparada com as taxas específicas da população de referência, para obtenção das Razões Padronizadas de Mortalidade por Câncer (SCMRs) segundo sítio anatômico da neoplasia, ajustadas por faixa etária e período de anos-calendário. Para cada SCMR, foi calculado um intervalo de confiança de 95\% (Checkoway et al., 1989).

A população do sexo masculino de vinte ou mais anos de idade, residente no Município do Rio de Janeiro, no período do estudo, foi escolhida como população de referência. A escolha dessa população deveu-se à disponibilidade de estimativas populacionais confiáveis para todo o período do estudo; à deficiência da qualidade das informações de mortalidade em pequenos municípios do interior do país, onde se situam unidades da empresa, ao percentual (36\%) de funcionários que trabalham na sede da empresa, naquele município; ao freqüente deslocamento de mão-de-obra da sede para outros municípios e estados; ao fato de as taxas de mortalidade por câncer do Município do Rio de Janeiro serem mais elevadas do que as de municípios do interior, o que poderia levar, no máximo, a uma estimativa conservadora dos resultados.

Os dados de população utilizados no estudo são estimativas anuais da população masculina residente no Município do Rio de Janeiro, por grupos de idade de cinco em cinco anos, dos 20 aos 69 anos, sendo o último grupo etário constituído por aqueles de setenta ou mais anos. Essas estimativas foram elaboradas pelo Instituto de Planejamento do Município do Rio de Janeiro (IPLAN-RIO, 1998), para o período 1978-1994.

Os dados de mortalidade foram obtidos através do CD-Rom do Sistema Brasileiro de Mortalidade do Ministério da Saúde (MS, 1996).
Todos os óbitos de indivíduos do sexo masculino, de vinte ou mais anos de idade, residentes no Município do Rio de Janeiro, foram transcritos para um banco de dados e agrupados, em nível de três dígitos, por grupos de causas de morte e, posteriormente, no caso das neoplasias, segundo as diferentes localizações. Para fins do cálculo das taxas de mortalidade por período de anos-calendário e faixa etária, esses óbitos foram agrupados em quatro períodos (1978-1979; 1980-1984; 1985-1989; 1990-1994) e onze faixas etárias (20-24 anos; 25-29 anos; 30-34 anos; 35-39 anos; 40-44 anos; 45-49 anos; 50-54 anos; 55-59 anos; 60-64 anos; 65-69 anos; 70 anos ou mais). Para o ano de 1978, só havia disponibilidade dos dados de mortalidade que constam das publicações do Sistema de Mortalidade (MS, 1979). Para os diferentes grupos de causas de morte, foram utilizados os dados publicados para o ano de 1978. Em relação às neoplasias, porém, só foram publicados os dados referentes a algumas localizações anatômicas. Nesse caso, optamos por utilizar, para o período 1978-1979, os dados referentes ao ano de 1979.

Em uma etapa posterior, foram realizadas comparações internas na coorte, através do cálculo de razões de taxas ajustadas (Breslow \& Day, 1987). A exposição do indivíduo foi determinada com base na sua ocupação e nos níveis operacionais da empresa. Mesmo levando em conta as limitações de uma medida indireta de exposição, julgamos pertinente utilizá-la, já que o objetivo do estudo era realizar uma análise exploratória da mortalidade por câncer neste grupo de trabalhadores.

Os trabalhadores foram classificados nos grupos superior, técnico e administrativo, de acordo com a ocupação que constava na folha de pagamento de dezembro de 1994, tendo como base uma relação fornecida pela empresa, com as ocupações pertencentes a cada um dos níveis. A análise de uma amostra aleatória de novecentos trabalhadores revelou que, no transcurso dos 17 anos do período de seguimento, ocorreram poucas mudanças de ocupação entre os diferentes níveis e/ou dentro do mesmo nível e, por esse motivo, optamos por utilizar apenas essa folha.

Na opinião de especialistas da empresa, apenas os trabalhadores do nível técnico estão sujeitos à exposição ocupacional a agentes cancerígenos. Não é possível, entretanto, analisar a exposição a cada um desses agentes de forma isolada, uma vez que o indivíduo exposto a um deles, em virtude de sua ocupação, também se encontra exposto aos outros. Não julgamos conveniente utilizar o nível superior como grupo não exposto, uma vez que o mesmo é cons- 
tituído por cerca de $50 \%$ de engenheiros, categoria profissional que a literatura tem mencionado como exposta a um nível mais elevado de radiação eletromagnética (Loomis \& Savitz, 1990; Olin, et al., 1985; Pearce et al., 1989).

Os trabalhadores administrativos foram considerados como grupo de referência (não exposto), sendo comparados com dois grupos diferentes de trabalhadores expostos: O primeiro grupo era formado pela categoria ocupacional "engenheiro"; o segundo era constituído pelos trabalhadores do nível técnico da empresa. A mortalidade por câncer desses dois grupos foi comparada com a do grupo não exposto, por meio do cálculo da razão de taxas, ajustada por faixa etária, período de anos-calendário e tempo de serviço. Para cada razão de taxas obtida, foi calculado um intervalo de confiança de 95\% (Breslow \& Day, 1987).

\section{Resultados}

Satisfizeram os critérios de inclusão 10.017 trabalhadores que formaram a coorte do estudo. Não foram obtidas informações sobre a condição vital de 299 trabalhadores, que representaram 3\% do total de indivíduos do estudo. Foi verificada a ocorrência de 498 óbitos (5\%).

A Tabela 2 mostra o número total de trabalhadores integrantes da coorte, a média de idade ao ingressar no estudo, e a média de tempo de serviço. A coorte de eletricitários pode ser caracterizada como um grupo formado por trabalhadores ainda jovens e com um tempo de serviço não muito longo. Nas categorias de tempo de serviço entre 5 e 14 anos, encontravam-se $43 \%$ dos trabalhadores.

Dos trabalhadores vivos ao término do estudo, 6.260 ainda se achavam em atividade na empresa $(67,9 \%)$ e 2.960 eram aposentados, num total de 9.220 indivíduos, o que corresponde a mais de $90 \%$ da coorte. Ocorreram 242 óbitos em trabalhadores ativos e 256 óbitos relativos a trabalhadores que haviam deixado a empresa. O maior número de óbitos ocorreu em faixas etárias ainda jovens (45 a 54 anos), e na categoria de 15 a 24 anos de serviço. As doenças do aparelho circulatório foram a causa de morte mais freqüente, correspondendo a um percentual de $35 \%$ do total de óbitos, vindo a seguir as causas externas $(21 \%)$. Os neoplasmas foram a terceira causa de óbito, sendo responsáveis por 96 mortes (19\% do total).

Entre as neoplasias, a causa de morte mais freqüente foi o câncer de pulmão (22\%) e, em segundo lugar, câncer de estômago (13\%). As neoplasias de localização não especificada representaram $6,2 \%$ do total de óbitos. Foi verificada a ocorrência de um número maior de óbitos na faixa etária de 45 a 59 anos e na categoria de 15 a 19 anos de trabalho na empresa.

A Tabela 3 apresenta as SMRs gerais, e por grupos de causas de morte, ajustadas por faixa etária e período de anos-calendário, para a coorte de eletricitários. A SMR para o conjunto de causas de morte mostra uma mortalidade menor na coorte de eletricitários. Da mesma forma, as SMRs para os diferentes grupos de causas de morte, apresentaram valores abaixo da unidade.

A Tabela 4 apresenta as SCMRs segundo localização anatômica das neoplasias, ajustadas por faixa etária e períodos de anos-calendário, com seus respectivos intervalos de confiança. Foi observado um aumento do número de óbitos para algumas localizações, como as neoplasias da vesícula biliar (SMR 2,64; IC 95\%: 0,997,03, com base em quatro óbitos observados), do pâncreas (SMR 1,83; IC 95\%: 0,91-3,66, oito óbitos observados), de cérebro (SMR 1,10; IC 95\%: 0,46-2,64, cinco óbitos observados) e os

Tabela 2

Coorte de trabalhadores eletricitários.

\begin{tabular}{|c|c|c|}
\hline Condição vital dos trabalhadores ao final do período de seguimento & $\mathbf{n}$ & $\%$ \\
\hline Vivos & 9.220 & 92,0 \\
\hline Mortos & 498 & 5,0 \\
\hline Ignorada & 299 & 3,0 \\
\hline Total & 10.017 & 100,0 \\
\hline Média de idade ao ingressar no estudo & \multicolumn{2}{|c|}{34,8 anos } \\
\hline Média de tempo de serviço & \multicolumn{2}{|c|}{15,5 anos } \\
\hline
\end{tabular}


Razões padronizadas de mortalidade (SMRs) por grupos de causas de morte e respectivos intervalos de confiança.

\begin{tabular}{lcccc}
\hline Grupos de causas de morte & $\begin{array}{c}\text { Óbitos } \\
\text { observados }\end{array}$ & $\begin{array}{c}\text { Óbitos } \\
\text { esperados }\end{array}$ & SMR & IC 95\% \\
\hline Doenças infecto-parasitárias & 13 & 45,5 & 0,29 & $0,17-0,50$ \\
Neoplasmas & 96 & 130,9 & 0,73 & $0,60-0,90$ \\
Doenças do sangue & 2 & 2,0 & 0,99 & $0,25-3,95$ \\
Doenças das glândulas endócrinas & 16 & 73,2 & 0,22 & $0,13-0,36$ \\
Doenças do sistema nervoso & 1 & 9,1 & 0,11 & $0,01-0,78$ \\
Doenças do aparelho circulatório & 176 & 334,9 & 0,52 & $0,45-0,60$ \\
Doenças do aparelho respiratório & 32 & 75,6 & 0,42 & $0,30-0,60$ \\
Doenças do aparelho digestivo & 28 & 87,7 & 0,32 & $0,22-0,46$ \\
Doenças do aparelho gênito-urinário & 4 & 11,5 & 0,35 & $0,13-0,93$ \\
Causas externas & 106 & 238,9 & 0,44 & $0,37-0,54$ \\
Todas as causas & 498 & $1.069,6$ & 0,47 & $0,43-0,51$ \\
\hline
\end{tabular}

IC $=$ Intervalo de Confiança.

Tabela 4

Razões padronizadas de mortalidade por câncer (SCMRs) segundo localização anatômica da neoplasia.

\begin{tabular}{|c|c|c|c|c|}
\hline Localização anatômica & $\begin{array}{c}\text { Óbitos } \\
\text { observados }\end{array}$ & $\begin{array}{c}\text { Óbitos } \\
\text { esperados }\end{array}$ & SCMR & IC $95 \%$ \\
\hline Cavidade oral/faringe (COO-C14) & 4 & 10,7 & 0,37 & $0,14-1,00$ \\
\hline Esôfago (C15) & 1 & 6,6 & 0,15 & $0,02-1,07$ \\
\hline Estômago (C16) & 13 & 14,1 & 0,92 & $0,53-1,59$ \\
\hline Intestino delgado (C17) & 3 & 0,3 & 10,35 & $3,34-32,09$ \\
\hline Cólon (C18) & 4 & 4,8 & 0,84 & $0,32-2,24$ \\
\hline Reto/Ânus (C20-C21) & 2 & 2,7 & 0,75 & $0,19-3,01$ \\
\hline Fígado (C22) & 2 & 4,2 & 0,48 & $0,12-1,91$ \\
\hline Vesícula biliar (C23) & 4 & 1,5 & 2,64 & $0,99-7,03$ \\
\hline Pâncreas (C25) & 8 & 4,4 & 1,83 & $0,91-3,66$ \\
\hline Aparelho digestivo (C15-C26) & 41 & 52 & 0,79 & $0,58-1,07$ \\
\hline Laringe (C32) & 1 & 5,6 & 0,18 & $0,02-1,27$ \\
\hline Pulmão (C34) & 20 & 30,1 & 0,66 & $0,43-1,03$ \\
\hline Aparelho respiratório (C30-C39) & 21 & 36,4 & 0,58 & $0,38-0,88$ \\
\hline Ossos (C40-C41) & 1 & 1,1 & 0,89 & $0,13-6,34$ \\
\hline Tecido conjuntivo (C45-C49) & 1 & 0,7 & 1,36 & $0,19-9,64$ \\
\hline Melanoma (C43) & 2 & 1,1 & 1,82 & $0,46-7,28$ \\
\hline Próstata (C61) & 2 & 5,4 & 0,37 & $0,09-1,48$ \\
\hline Bexiga (C67) & 4 & 2,6 & 2,60 & $0,58-4,12$ \\
\hline $\operatorname{Rim}(\mathrm{C} 64-\mathrm{C} 65)$ & 7 & 2.4 & 2,91 & $1,39-6,10$ \\
\hline Cérebro (C71) & 5 & 4,5 & 1,10 & $0,46-2,64$ \\
\hline Glândulas endócrinas (C73-C75) & 1 & 0,6 & 1,64 & $0,23-11,65$ \\
\hline Linfomas (C82-C85) & 3 & 2,9 & 1,02 & $0,33-3,17$ \\
\hline Leucemias (C91-C95) & 2 & 4,6 & 0,44 & $0,11-1,75$ \\
\hline
\end{tabular}

IC = Intervalo de Confiança. 
melanomas (SMR 1,82; IC 95\%: 0,46-7,28, dois óbitos observados). Esses resultados, entretanto, englobam a unidade nos seus intervalos de confiança.

Com relação às neoplasias de fígado, leucemias e linfomas, as SMRs obtidas alcançaram valores inferiores a 1,0.

Foi observado um aumento dos óbitos por neoplasias de rim (SMR 2,91; IC 95\%: 1,39-6,10, com base em sete óbitos observados) e de intestino delgado (SMR 10,35; IC 95\%: 3,34-32,09, três óbitos observados), ambos estatisticamente significativos.

A Tabela 5 apresenta as razões de taxas, ajustadas por faixa etária, período de anos-calendário e tempo de serviço, para o conjunto de engenheiros e para o grupo de nível técnico, tendo como referência os trabalhadores administrativos. Para os engenheiros, essa medida apresenta valor semelhante à unidade, não configurando um risco aumentado de desenvolvimento de neoplasias para esse grupo ocupacional. Observamos um maior risco de desenvolvimento de neoplasias para o grupo técnico em relação aos funcionários administrativos, embora o resultado não seja estatisticamente significativo. Esses resultados praticamente não se modificaram quando foi realizada a análise isolada, por faixa etária, período de anos-calendário e tempo de serviço, razão pela qual só foi apresentado o resultado ajustado.

\section{Discussão}

Embora a validade da SMR seja alvo de muito debate, ela tem sido utilizada em um grande número de estudos epidemiológicos, para analisar o padrão de mortalidade de coortes ocupacionais (Band et al., 1996; Rivard et al., 1994; Stayner et al., 1993). Estudos exploratórios utilizam a SMR como técnica de análise, com bastante freqüência, pois essa medida permite um rastreamento das causas de óbito que apresen- tam maior mortalidade na população de estudo (Breslow \& Day, 1987).

A análise da SMR para todas as causas de morte revelou uma diminuição estatisticamente significativa da mortalidade na coorte de eletricitários. Da mesma forma, as SMRs para os diferentes grupos de causas de morte mostraram um menor número de óbitos entre os eletricitários, e seus intervalos de confiança, com exceção da SMR para doenças do sangue, não incluem a unidade.

Alguns autores destacam a importância das diferenças de mortalidade entre classes sociais e diferentes ocupações, atribuindo a elas modificações observadas no efeito do trabalhador sadio (Howe et al., 1988; Wilcosky \& Wing, 1987). Outros pesquisadores atribuem essas diferenças às formas de seleção para o trabalho, considerando que, quanto melhor o emprego, em termos de remuneração e local de trabalho, mais rigoroso será o processo seletivo (McMichael et al., 1975; Monson, 1986). A realização desse estudo em uma empresa onde existem melhores condições de trabalho e os salários situam-se em patamares mais elevados do que aqueles do conjunto da população nos leva a supor que uma parte substancial do efeito do trabalhador sadio observado possa estar refletindo essas diferenças. Por outro lado, acompanhando o emprego, surgem mudanças no estilo de vida e há uma maior facilidade de acesso a atendimento médico, fatores que podem contribuir para a diminuição do número de óbitos entre os trabalhadores. Dentro da mesma empresa, a ocupação, por si só, já constitui um aspecto determinante do nível sócioeconômico e, em conseqüência, de diferenças na mortalidade.

A coorte deste estudo é formada por trabalhadores ativos e inativos, o que, segundo a literatura, tenderia a diminuir o efeito do trabalhador sadio (Howe et al., 1988). Essa diminuição parece não ter acontecido, provavelmente porque as diferenças sócio-econômicas entre

Tabela 5

Razão de taxas de Mantel-Haenszel para neoplasias (engenheiros e trabalhadores do grupo técnico)

\begin{tabular}{lcccc}
\hline Tipo de trabalho & $\mathbf{n}$ & Óbitos & $\begin{array}{c}\text { Razão de taxas de } \\
\text { Mantel-Haenszel }\end{array}$ \\
\hline Técnicos & & 4.294 & $45 \%$ & 1,30 \\
Engenheiros & 1.616 & 13 & 1,02 & $0,81-2,07$ \\
Administrativos & 2.494 & 29 & - & - \\
\hline
\end{tabular}


os eletricitários e a população de referência tiveram um maior peso, já que muitos benefícios recebidos pelos trabalhadores ativos são mantidos na aposentadoria. Uma diferença acentuada entre as condições de saúde da coorte ocupacional e da população de referência pode mascarar os resultados de um estudo, deixando de apontar a existência de riscos aumentados para determinadas doenças, mesmo que esses estejam presentes.

A causa básica de morte também exerce papel importante sobre o efeito do trabalhador sadio. Esse efeito tende a ser mais acentuado em doenças que aparecem em idades jovens, impedindo a participação do indivíduo na força de trabalho, ou que interferem na atividade laboral (Monson, 1986; Weed et al., 1987; Wen et al., 1983). Com relação à mortalidade por neoplasias, existe consenso na literatura de que essa costuma ser menos afetada pelo efeito do trabalhador sadio, pois não parece existir uma forma de seleção que possa identificar os indivíduos que virão a desenvolver a doença (Monson, 1986; Weed et al., 1987). Assim, a análise da SCMR tende a apresentar resultados mais fidedignos do que aqueles observados para os demais grupos de causas de morte.

Na coorte de eletricitários, com exceção das doenças do sangue, cuja SMR foi baseada em um número muito pequeno de óbitos, a SMR para neoplasias apresentou o maior valor, embora o mesmo tenha se situado, como os demais, abaixo da unidade. Entretanto, a hipótese desse estudo, em consonância com a literatura, não era a de observar um maior número de óbitos por todos os tipos de câncer, considerados em conjunto, mas sim um aumento da mortalidade por alguns tipos específicos, associados aos fatores de risco presentes no ambiente ocupacional do setor elétrico.

Vale a pena lembrar, também, a heterogeneidade do grupo de eletricitários, composto tanto por indivíduos expostos aos fatores de risco analisados, como por indivíduos não expostos, fato que tende a conduzir as estimativas de risco em direção à unidade (Copeland et al., 1977; Flegal et al., 1986). Além disso, o próprio método de cálculo da SMR faz com que essa medida represente uma média dos resultados observados entre os diferentes subgrupos que constituem a coorte e, como tal, diferenças para mais, ou para menos, são canceladas umas pelas outras (Breslow \& Day, 1987). É necessário lembrar, ainda, o pequeno número de óbitos por cada tipo específico de câncer observado na coorte de eletricitários.

Diversos estudos epidemiológicos observaram associação entre a exposição a campos eletromagnéticos e o desenvolvimento de leucemias neste grupo de trabalhadores. Na coorte de eletricitários eram esperados 4,6 óbitos por leucemia, tendo ocorrido somente dois, o que resultou em uma SCMR de 0,44, com intervalo de confiança incluindo a unidade. Esse resultado é semelhante ao observado em outro estudo realizado no Brasil (Mattos \& Koifman, 1996).

Em relação às neoplasias de cérebro, outra localização anatômica para a qual tem sido observada uma associação com a exposição ocupacional aos campos eletromagnéticos, foi encontrada, neste estudo, uma SCMR de 1,10, cujo intervalo de confiança incluiu a unidade. No outro estudo brasileiro, havia sido observado um excesso de óbitos por essa neoplasia entre os trabalhadores cujas ocupações envolviam uma exposição provavelmente elevada aos campos magnéticos (Mattos \& Koifman, 1996).

Os resultados do presente estudo mostraram uma SCMR acima de 1,0 para os melanomas, mas o intervalo de confiança inclui a unidade e o resultado é baseado na ocorrência de dois óbitos, contra 1,1 esperado.

A associação entre exposição aos campos eletromagnéticos e linfomas, observada na literatura, não foi encontrada na coorte de estudo, onde o número de óbitos observados foi praticamente semelhante ao número de óbitos esperados, com base nas taxas de mortalidade da população de referência.

As localizações anatômicas que têm sido associadas à exposição a PCBs são os tumores de fígado, vesícula biliar, cérebro e melanomas. Neste estudo, foi observado um excesso de óbitos por neoplasias da vesícula biliar (quatro óbitos observados/1,5 esperado). Embora o intervalo de confiança desse resultado tenha incluído a unidade, seu limite inferior está bastante próximo da mesma, e o número de óbitos observados é bastante superior ao número esperado. Seria interessante o seguimento dessa coorte por um período de tempo maior, para avaliar o comportamento da mortalidade por essa neoplasia.

Quanto aos solventes, os resultados do presente estudo, em relação a leucemias e tumores de cérebro, foram comentados anteriormente.

Existem poucas evidências, na literatura, sobre a associação entre exposição a campos eletromagnéticos e o desenvolvimento de tumores do aparelho urinário. Na coorte de eletricitários foi observada uma SCMR de 2, 91 (IC 95 \%: 1,39-6,10) para câncer de rim (sete óbitos observados /2,4 esperados). Em relação a câncer de bexiga, foi verificado também um aumento do número de óbitos (quatro óbitos ob- 
servados/2,6 esperados), mas o intervalo de confiança incluiu a unidade. No outro estudo com eletricitários realizado no Brasil, foi observado um aumento do número de óbitos por câncer de bexiga entre os trabalhadores com exposição provavelmente alta aos campos magnéticos (Mattos \& Koifman, 1996).

Verificou-se um aumento da mortalidade por tumores do intestino delgado entre os eletricitários (SCMR 10,35; IC 95\%: 3,34-32,09). Essa neoplasia não constituiu uma hipótese de investigação do estudo e, na literatura, não existe menção sobre sua ocorrência em relação aos agentes cancerígenos analisados. $\mathrm{O}$ resultado obtido, embora estatisticamente significativo, é baseado em um número pequeno de óbitos (três observados/0,3 esperado). Todavia, em virtude da magnitude da SCMR, seria interessante que esse tumor fosse analisado em outros estudos epidemiológicos.

Os resultados das comparações internas realizadas neste estudo não mostraram um maior risco de desenvolvimento de câncer para os engenheiros, em comparação com os trabalhadores administrativos. Em relação aos técnicos, foi observada uma razão de taxas um pouco acima de 1,0, mas o intervalo de confiança engloba a unidade. Ressaltamos, mais uma vez, que a ocorrência de um maior risco de desenvolvimento do conjunto de neoplasias não constituiu uma hipótese do presente estudo e que a categoria de trabalhadores agrupada como "nível técnico" consiste em um grupo bastante heterogêneo de indivíduos expostos e não expostos, o que tende a subestimar as estimativas de risco obtidas. O número limitado de óbitos por neoplasias observados na coorte não permitiu, contudo, a realização de análises por tipos específicos de câncer ou em subgrupos mais homogêneos em termos de exposição.

A qualidade dos dados provenientes de atestados de óbito, como material para estudos epidemiológicos, tem sido analisada por diversos pesquisadores, que destacam a maior confiabilidade da classificação da causa básica de morte para casos de câncer (Barclay \& Phillips, 1962; Monteiro et al., 1994; Percy et al., 1981). Não temos informação sobre o quantitativo de tumores confirmado por diagnóstico histopatológico, sendo possível que alguns tipos específicos de neoplasias tenham sido classificados de maneira equivocada. Da mesma forma, é desconhecido o percentual de confirmação histopatológica para o conjunto de óbitos por neoplasias da população de referência, mas não há nenhum motivo para supor que isso ocorra de uma maneira sistemática, ou que ambos sejam muito diferentes.
Uma debilidade deste trabalho consiste na determinação da exposição. A utilização de categorias ocupacionais como medida indireta de exposição tem sido bastante criticada na literatura (Coleman \& Beral, 1988; Savitz et al., 1993). Nos últimos anos, entretanto, a disponibilidade de dosímetros para medir campos elétricos e magnéticos tem permitido definir, com maior segurança, a exposição ocupacional das diferentes categorias de eletricitários (Deadman et al., 1991).

Outra limitação deste estudo consiste na falta de informação sobre variáveis de confundimento, presentes no ambiente de trabalho, ou relacionadas ao estilo de vida dos indivíduos, entre elas o tabagismo e o álcool, já firmemente estabelecidos na literatura como fatores de risco para câncer.

A ausência de informação sobre tabagismo no presente trabalho não permite uma análise isolada de seu efeito, independentemente dos fatores de risco que estão sendo estudados. Entretanto, as SMRs observadas para doenças do aparelho respiratório e câncer do aparelho respiratório foram menores do que a unidade e, estatisticamente significativas, o que pode ser um indicativo de que a prevalência de tabagismo, neste grupo de trabalhadores, não seja maior do que na população utilizada como referência.

Da mesma forma, não foi possível analisar a influência do consumo de álcool nos resultados obtidos neste estudo. A SMR para doenças do aparelho digestivo, evidenciando um menor número de óbitos por essa causa de morte na coorte de eletricitários, em comparação com a população, pode, novamente, sugerir que a prevalência desse fator de risco entre seus membros não seja mais elevada do que na população masculina do Rio de Janeiro.

Uma limitação importante desse estudo é o fato de a coorte ser constituída por indivíduos ainda jovens, grande parte dos quais, ao final do período de seguimento, não tinha ainda atingido a idade de 55 anos. A formação da coorte, limitada aos trabalhadores em atividade no ano de 1978 ou admitidos a partir dessa data, contribuiu para a exclusão dos trabalhadores mais antigos e, portanto, mais velhos, uma vez que os mesmos já haviam se aposentado na época de início do estudo. Em decorrência disso, $43 \%$ dos trabalhadores membros da coorte tinham menos de 15 anos de serviço, o que representa, indiretamente, uma exposição cumulativa menor aos fatores de risco ocupacionais. Além disso, os diferentes tipos de câncer analisados se caracterizam por apresentar períodos de latência razoavelmente lon- 
gos, após a exposição. Esses fatos contribuíram para diminuir o número de óbitos acumulados ao final do período de seguimento. O número limitado de óbitos observados por cada localização anatômica das neoplasias não foi suficiente para propiciar estabilidade aos resultados da análise estatística, o que se evidenciou em intervalos de confiança amplos para quase todas as SCMRs. Além disso, o fato de haver muitos sítios anatômicos de câncer tornou necessário calcular um grande número de SCMRs, aumentando a probabilidade da ocorrência de resultados positivos, unicamente devidos ao acaso.

Esse estudo constitui um primeiro passo para a análise das condições de saúde dos eletricitários. Por causa das limitações já colocadas, seus resultados são de difícil interpreta- ção, à luz do conhecimento disponível sobre o tema, e, em conseqüência, não têm aplicabilidade direta, em termos de prevenção de câncer, nesse grupo de trabalhadores.

Do ponto de vista da saúde pública, a importância de determinar se a relação entre exposição a campos eletromagnéticos e a ocorrência de câncer é de natureza causal está relacionada ao número de indivíduos expostos a essa radiação que, nos tempos atuais, abrange, praticamente, o conjunto da população. Esse fato confere um risco atribuível potencial bastante elevado a essa possível associação. Nesse sentido, a ampliação do conhecimento dessa área, no ambiente ocupacional, acarretaria não só a adoção de medidas voltadas para a proteção dos trabalhadores, mas também para toda a população.

\section{Referências}

BAND, P. R.; LE, N. D.; FANG, R.; DESCHAMPS, M.; COLDMAN, A. J.; GALLAGHER, R. P. \& MOODY, J., 1996. Cohort study of Air Canada pilots: Mortality, cancer incidence and leukemia risk. American Journal of Epidemiology, 143:137-143.

BARCLAY, T. H. C. \& PHILLPS, A. J., 1962. The accuracy of cancer diagnosis on death certificates. Cancer, 15:5-9.

BASTUJI-GARIN, S.; RICHARDSON, S. \& ZITTOUN, R., 1990. Acute leukaemia in workers exposed to electromagnetic fields. European Journal of Cancer, 26:1119-1120.

BORLAND INTERNATIONAL, 1986. DBase III. Scotts Valley: Borland International.

BRESLOW, N. E. \& DAY, N. E., 1987. Statistical Methods in Cancer Research. Volume II: The Design and Analysis of Cohort Studies. IARC Scientific Publications 82. Lyon: International Agency for Research on Cancer.

BROWN, D. P., 1987. Mortality of workers exposed to polychlorinated biphenyls - An update. Archives of Environmental Health, 42:333-339.

CDC (Centers for Disease Control and Prevention) / WHO (World Health Organization), 1996. Epi Info 6, Version 6.04. A Word Processing Database, and Statistics Program for Public Health. Atlanta: CDC/Geneva: WHO.
CHECKOWAY, H.; PEARCE, N. \& CRAWFORD-BROWN, D. J., 1989. Research Methods in Occupational Epidemiology. New York: Oxford University Press.

COLEMAN, M. \& BERAL, V., 1988. A review of epidemiological studies of the health effects of living near or working with electricity generation and transmission equipment. International Journal of Epidemiology, 17:1-13.

COPELAND, K. T.; CHECKOWAY, H.; McMICHAEL, A. J. \& HOLBROOK, R. H., 1977. Bias due to misclassification in the estimation of relative risk. American Journal of Epidemiology, 105:488-495.

DEADMAN, J. E.; SCHNITMAN, A. \& THÉRIAULT, G., 1991. Reconstruction of Past Exposures to Electric and Magnetic Fields. Montreal: Joint Électricité de France/Hydro-Québec/Ontario Hydro. (mimeo.)

FERNICOLA, N. G. G.; WAKAMATSU, C. T.; MENDES, R. \& MORAES, E. C. F., 1976. Urinary output of phenol in workmen exposed to benzene in the shoe industry. Revista de Saúde Pública, 10:327333.

FLEGAL, W. D.; BROWNIE, C. \& HAAS, J. D., 1986. The effects of exposure misclassification on estimates of relative risks. American Journal of Epidemiology, 123:736-751.

FLODIN, U.; FREDRIKSSON, M.; AXELSON, A; PERSSON, B. \& HARDELL, L., 1986. Background radia- 
tion, electrical work, and some other exposures associated with acute myeloid leukemia in a casereferent study. Archives of Environmental Health, 41:77-84.

GRAYSON, J. K., 1996. Radiation exposure, socioeconomic status, and brain tumor risk in the US Air Force: A nested case-control study. American Journal of Epidemiology, 143:480-486.

HOWE, G. R.; CHIARELLI, A. M. \& LINDSAY, J. P., 1988. Components and modifiers of the healthy worker effect: Evidence from three occupational cohorts and implications for industrial compensation. American Journal of Epidemiology, 128: 1364-1375.

IPLAN-RIO (Instituto de Planejamento do Município do Rio de Janeiro), 1998. Estimativas de População para o Rio de Janeiro. Rio de Janeiro: IPLANRIO. (mimeo.)

KATO, M.; ROCHA, M. L. R.; CARVALHO, A. B.; CHAVES, M. E. C.; RANA, M. C. M. \& OLIVEIRA, F. C., 1993. Occupational exposure to neurotoxicants; preliminary survey in five industries of the Camaçari petrochemical complex, Brazil. Environmental Research, 61:133-139.

KOIFMAN, S. \& THÉRIAULT, G., 1994. Electric and magnetic fields and cancer: The use of field exposure measurements in epidemiological studies. In: Biological Effects of Electric and Magnetic Fields (D. O. Carpenter \& S. Ayrapetyan, ed.), v. 2, pp. 201-231, San Diego: Academic Press.

LAZAROV, D.; WALDRON, H. A. \& PEJIN, D., 2000. Acute myeloid leukaemia and exposure to organic solvents - A case-control study. European Journal of Epidemiology, 16:295-301.

LIN, R. S.; DISCHINGER, P. C.; CONDE, J. \& FARREL, K. P., 1985. Occupational exposure to electromagnetic fields and the occurrence of brain tumors. Journal of Occupational Medicine, 27:413-419.

LOOMIS, D. P. \& SAVITZ, D. A., 1990. Mortality from brain cancer and leukemia among electrical workers. British Journal of Industrial Medicine, 47:633638.

MATTOS, I. E. \& KOIFMAN, S., 1996. Mortalidade por câncer em trabalhadores de companhia geradora de eletricidade do Estado de São Paulo, Brasil. Revista de Saúde Pública, 30:564-575.

McMICHAEL, A. J.; HAYNES, S. G. \& TYROLER, H. A., 1975. Observations on the evaluation of occupational mortality data. Journal of Occupational Medicine, 17:128-131.

MILHAM Jr., S., 1982. Mortality from leukemia in workers exposed to electrical and magnetic fields. New England Journal of Medicine, 307:249.

MILHAM Jr., S., 1985. Mortality from leukemia in workers exposed to electromagnetic fields. Environmental Health Perspectives, 62:297-300.

MONSON, R. R., 1986.Observations on the healthy worker effect. Journal of Occupational Medicine, 28:425-437.

MONTEIRO, G. T. R.; KOIFMAN, R. J. \& KOIFMAN, S., 1997. Confiabilidade e validade dos atestados de óbito por neoplasias. I. Confiabilidade da codificação para o conjunto das neoplasias no Estado do Rio de Janeiro. Cadernos de Saúde Pública, 13 (Sup. 1):53-65.

MS (Ministério da Saúde), 1979. Estatísticas de Mor- talidade, Brasil 1978. Brasília: MS.

MS (Ministério da Saúde), 1996. Estatísticas de Mortalidade, Brasil 1979-1994. CD-ROM. Brasília: MS.

OLIN, R.; VAGERO, D. \& AHLBOM, A., 1985. Mortality experience of electrical engineers. British Journal of Industrial Medicine, 42:211-212.

OMS (Organização Mundial da Saúde), 1985. Manual da Classificação Estatística Internacional de Doenças, Lesões e Causas de Óbitos - Nona Conferência de Revisão. São Paulo: Centro Brasileiro de Classificação de Doenças em Português.

OUW, H. K.; SIMPSON, G. R.\& SIYALI, D. S., 1976. Use and health effects of Aroclor 1242, a polychlorinated biphenyl, in an electrical industry. Archives of Environmental Health, 31:189-194.

PEARCE, N.; REIF, J. \& FRASER, J., 1989. Case-control studies of cancer in New Zealand electrical workers. International Journal of Epidemiology, 18:5559.

PERCY, C.; STANEK E. \& GLOECKER, L., 1981. Accuracy of cancer death certificates and its effect on cancer mortality statistics. American Journal of Public Health, 71:242-250.

PHILLIPS, D. L.; SMITH, A. B.; BURSE, V. W.; STEELE, G. K.; NEEDHAM, L. L. \& HANNON, W. H., 1989. Half-life of polychlorinated biphenyls in occupationally exposed workers. Archives of Environmental Health, 44:351-354

RIVARD, C. I.; DUFRESNE, A.; ARMSTRONG, B.; BOUCHARD, P. \& THÉRIAULT, G., 1994. Cohort study of silicon carbide production workers. American Journal of Epidemiology, 140:1009.

SAHL, J. D.; KELSH, M. A. \& GREENLAND, S., 1993. Cohort and nested case-control studies of hematopoietic cancers and brain cancer among electric utility workers. Epidemiology, 4:104-114.

SATIN, K. P.; WONG, O.; YUAN, L. A.; BAILEY, W. J.; NEWTON, K. L.; WEN, C. \& SWENCICKI, R. R., 1996. A 50-year mortality follow-up of a large cohort of oil refinery workers in Texas. Journal of Occupational Medicine, 38:492-506.

SAVITZ, D. A. \& LOOMIS, D. P., 1995. Magnetic field exposure in relation to leukemia and brain cancer mortality among electric utility workers. American Journal of Epidemiology, 141:123-134.

SAVITZ, D. A.; PEARCE, N. E. \& POOLE, C., 1993. Update on methodological issues in the epidemiology of electromagnetic fields and cancer. Epidemiologic Reviews, 15:558-566.

SCHREIBER, G. H.; SWAEN, G. M. H.; MEIJERS, J. M. M.; SLANGEN, J. J. M. \& STURMANS, F., 1993. Cancer mortality and residence near electricity transmission equipment: A retrospective cohort study. International Journal of Epidemiology, 22:9-15.

SINKS, T.; STEELE, G.; SMITH, A. B.; WATKINS, K. \& SHULTS, R. A., 1992. Mortality among workers exposed to polychlorinated biphenyls. American Journal of Epidemiology, 136:389-398.

STATA CORPORATION, 1997. Stata Statistical Software. Release 5.0. College Station: Stata Corporation.

STAYNER, L.; STEENLAND, K.; GREIFE, A.; HORMUNG, R.; HAYES, R.; NOWLIN, S.; MORAWETZ, J.; RINGENBURG, V.; ELLIOT, L. \& HALPERIN, W., 1993. Exposure-response analysis of cancer mor- 
tality in a cohort of workers exposed to ethylene oxide. American Journal of Epidemiology, 133:787798.

STEENLAND, K. \& STAYNER, L., 1991. The importance of employment status in occupational cohort mortality studies. Epidemiology, 2:418-423.

STERN, F. B.; WAXWEILER, R. A.; BEAUMONT, J. J.; LEE, S. T.; RINSKY, R. A.; ZUMWALDE, R. D.; HALPERIN, W. E.; BIERBAUM, P. J.; LANDRIGAN, P. J. \& MURRAY, J. R. W. E., 1986. A case-control study of leukemia at a naval nuclear shipyard. American Journal of Epidemiology, 123:980-992.

THÉRIAULT, G.; GOLDBERG, M.; MILLER, A. B.; ARMSTRONG, P.; GUÉNEL, J.; DEADMAN, J.; IMBERNON, E.; TO, T.; CHEVALIER, A.; CYR, D. \& WALL, C., 1994. Cancer risks associated with occupational exposure to magnetic fields among electric utility workers in Ontario and Quebec, and France: 1970-1989. American Journal of Epidemiology, 139:550-572.

THOMAS, T. L.; STOLLEY, P. D.; STEMHAGEN, A.; FONTHAM, E. T. H.; BLEECKER, M. L.; STEWART, P. A. \& HOOVER, R. N., 1987. Brain tumor mortality risk among men with electrical and electronics jobs: A case-control study. Journal of the $\mathrm{Na}$ tional Cancer Institute, 79:233-238.

TORNQVIST, S.; NORELL, S.; AHLBOM, A. \& KNAVE, B., 1986. Cancer in the electric power industry. British Journal of Industrial Medicine, 43:212-213.

TYNES, T.; ANDERSEN, A. \& LANGMARL, F., 1992. Incidence of cancer in Norwegian workers potentially exposed to electromagnetic fields. American Journal of Epidemiology, 136:81-88.

TYNES, T.; JYNGE, H. \& VISTNES, A. I., 1994. Leukemia and brain tumors in Norwegian railway workers: A nested case-control study. American Journal of Epidemiology, 139:645-653.
VAGERO, D.; AHLBOM, A.; OLIN, R. \& SAHLSTEN, S. 1985. Cancer morbidity among workers in the telecommunications industry. British Journal of Industrial Medicine, 40:191-195.

VAINIO, H.; MATOS, E. \& KOGEVINAS, M., 1994. Identification of occupational carcinogens. In: Occupational Cancer in Developing Countries $(\mathrm{N}$. Pearce, E. Matos, H. Vainio, P. Boffetta \& M. Kogevinas, ed.), IARC Scientific Publications 129, pp. 41-59, Lyon: International Agency for Research on Cancer.

WEED, D. L.; TYROLER, H. A. \& SHY, C., 1987. The healthy worker effect in actively working communications workers. Journal of Occupational Medicine, 29:335-339.

WEN, C. P.; TSAI, S. P. \& GIBSON, R. L., 1983. Anatomy of the healthy worker effect: A critical review. Journal of Occupational Medicine, 25:283-289.

WERTHEIMER, N. \& LEEPER, E., 1982. Adult cancer related to electrical wires near the home. International Journal of Epidemiology, 11:345-355.

WILCOSKY, T. \& WING, S., 1987. The health worker effect. Scandinavian Journal of Work and Environmental Health, 13:70-72.

WÜNSCH FILHO, V.; MAGALDI, C.; NAKAO, N. \& MONCAU, J. E. C., 1995. Trabalho industrial e câncer de pulmão. Revista de Saúde Pública, 29:166-176.

Recebido em 6 de dezembro de 2000

Versão final reapresentada em 18 de maio de 2001

Aprovado em 1 de agosto de 2001 